\title{
Citizen science for pedestrian cartography: collection and moderation of walkable routes in cities through mobile gamification
}

\author{
Ippokratis Kapenekakis ${ }^{*}$ [i] and Konstantinos Chorianopoulos (1)
}

\author{
*Correspondence: \\ ippokratis1@gmail.com \\ School of Science \& \\ Technology, Hellenic Open \\ University, Aristotelous 18, \\ 26335 Patra, Greece
}

\begin{abstract}
Digital geographical maps can be regarded as a user interface for understanding and navigating the city. Nevertheless, contemporary digital maps over-emphasize the needs of motor vehicles. Pedestrian routes have only been considered as an addon option in existing digital maps and the respective data collection has not been performed in the field. In this article, we present a mobile application that employs gamification as a means to engage users to collect pragmatic data about walkable routes, which are then processed with the goal of creating a new kind of pedestrianfriendly cartography. Besides the technical infrastructure for collecting and filtering the route traces, the main challenge in user-generated walkable routes is the identification of malicious data, which should be rejected, as well as the rewarding of constructive behavior through peer-review. For this purpose, the mobile application employs a point system in order to identify and discourage the submission of bad routes. $A$ longitudinal (2-months) field study of the mobile application confirmed that gamification facilitates effective data collection for producing pedestrian cartography even with few users and demonstrated that gamification infuses a shared responsibly about the quality of data collection. The visualization of the pedestrian-generated data on a map required a partially manual process, thus, further research should explore the gamification of the data visualization part, too.
\end{abstract}

Keywords: Gamification, Mapmaking, Cartography, Pedestrian, Route, Citizen science, Mobile App, Crowdsourcing

\section{Background}

The visibility of walkable routes in geographical maps is strongly associated with better well-being and higher quality of life in cities $[1,2]$. Pedestrians usually employ mapping and navigation applications (Apps) on their smart phones in order to identify and follow walkable paths in cities. In this way, digital maps can be regarded as an important user interface for understanding and navigating the city. Even though modern digital maps illustrate the real world in elaborate detail, they put emphasis on motor cars. Therefore, digital maps usually have a dominant aesthetic that serves navigation for motor drivers and makes all the maps to look the same [3]. Navigation Apps offer an option for

(c) The Author(s) 2017. This article is distributed under the terms of the Creative Commons Attribution 4.0 International License (http://creativecommons.org/licenses/by/4.0/), which permits unrestricted use, distribution, and reproduction in any medium, provided you give appropriate credit to the original author(s) and the source, provide a link to the Creative Commons license, and indicate if changes were made. 
pedestrians, but the respective algorithms prioritize distance rather than route walkability, because geographical accuracy is the main data point in their design and motor-vehicles their main target audience [4]. Indeed, Wilmott, in ethnographic research he did, revealed several missing components of navigation applications, resulting in unwillingness of pedestrians to fully engage with the application [5]. Therefore, it is likely that the main user interface employed by many pedestrians for understanding and navigating the city is not suitable for their particular needs.

For example, a digital map might depict two adjacent and parallel streets as having the same width and the respective navigation system might guide a pedestrian through any of the two, despite the major differences in actual walkability (Fig. 1). Indeed, in terms of car navigation, these two streets are exactly similar, since they have one lane for road traffic, but this kind of vehicle-centered information (visual or navigation instructions) is mostly irrelevant for pedestrians. In this work, we suggest that mobile navigation Apps could be improved by an emphasis on the needs and preferences of pedestrians. For this purpose, we have developed a mobile App that facilitates the creation of a pedestriangenerated street network on a digital map. In particular, we suggest that the street network layer could become more relevant to pedestrians, if the way of collecting route data is based on actual pedestrian behavior. In fact, the process in which Spatial Big Data collected is so important for the subsequent development of navigation applications and maps that use them, which, for example, led Wilmott in ethnographic research to understand the process [5].

Many people in urban areas always carry a smart phone, which is enhanced with accurate geo-location sensors and on-line services. Therefore, it is possible that the paths actually walked by the citizens to be (voluntarily) recorded by their mobile phone and shared with others in order to aggregate the collective intelligence about walkable routes. For this purpose, we created a mobile App that collects route data for pedestrian mapmaking. Since a mobile App collects data from many users, it enables cost-effective pedestrian mapmaking, but, at the same time, it raises the issue of motivating data collection and ensuring data quality. In this article, we explore some well-established

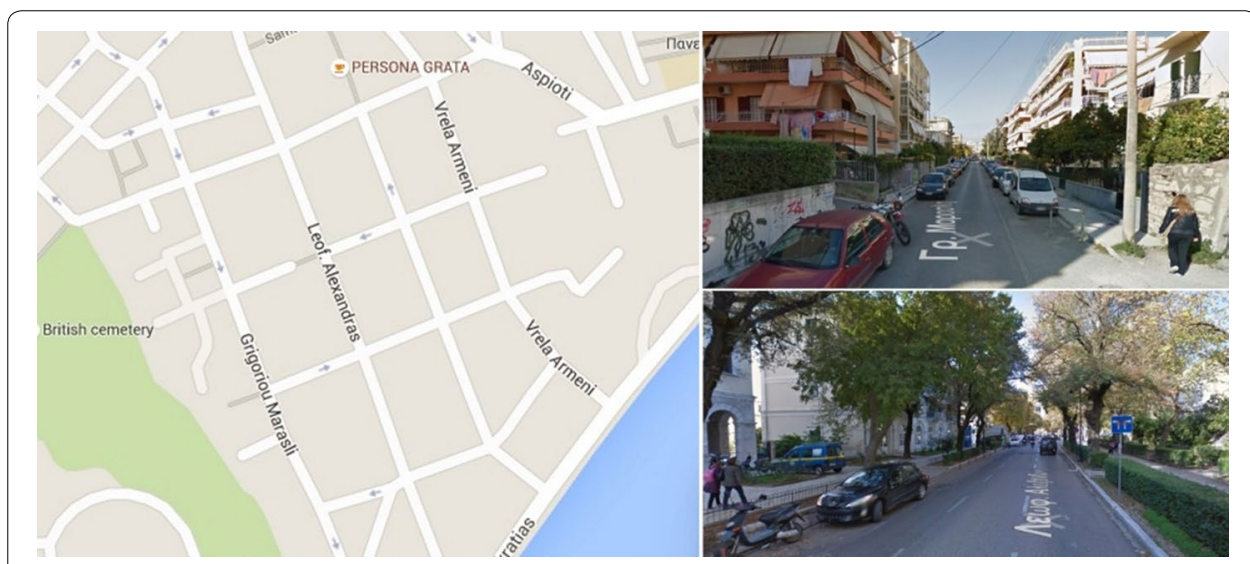

Fig. 1 Two adjacent and parallel streets 'Grigoriou Marasli' and 'Leof. Alexandras' are visually depicted as having the exact same width on a popular digital map (left), which is widely used on mobile Apps for navigation. Although these streets are depicted with the same width on the digital map and might be suggested for pedestrian navigation as similar options they are significantly different in walkability (right), with the latter street being very pleasant (bottom right) 
citizen-science challenges in the design and deployment of a mobile App for pedestrians. In particular, we consider the research question: "Does mobile gamification enable effective data collection for pedestrian cartography?" Our App employees peer-review to reward-ban good-bad behavior. Moreover, we validated the design with a field study that resulted into a pedestrian-centered map that is significantly different to the original vehicle-centered one.

The rest of this article is organized as follows: In "Related work" section, we provide an overview of mapmaking, as well as relevant mobile Apps. In "Mobile application design and implementation" section, we describe the design of the mobile App, as well as the gamification and peer-review sub-system of the App. In "Results" section, we demonstrate the App and the results from a longitudinal field study. In "Discussion" section, we provide a discussion about pedestrian mapmaking with a mobile App system, and about citizen science and gamification. Finally, in "Conclusion" section, we provide the conclusion of our work.

\section{Related work}

\section{Mapmaking and walkability}

Mapmaking could be defined as the study and practical making of maps [6]. Nevertheless, it is impossible to define objectively the best-designed map. In contrast, users' needs, tasks, and goals play an important role in the design of geographical maps. A map should include only the information that has a real meaning for the users' task. Also, a map shouldn't illustrate reality in too much detail [7]. Therefore, every map aiming to be used for walking in a city should aim at the needs of users and their subjective perception about the urban area [4]. In some cases, an abstract map might be more useful than a realistic one. For example, Coulton et al. [3] used abstraction of features on a digital map of a location based game in order to encourage "head-up" navigation, which contributed to the engagement of players with their natural surroundings. In another example, the London Tube map was improved by focusing on the needs of commuters in comparison to the original, which was geographically accurate (Fig. 2). Therefore, focusing on the needs of map users is more important than producing a geographically accurate map. Indeed, current digital maps focus very well on the needs of vehicle users, because they represent accurately the width of the streets, with respect to vehicle accessibility, but at the same time they neglect other users, such as pedestrians.

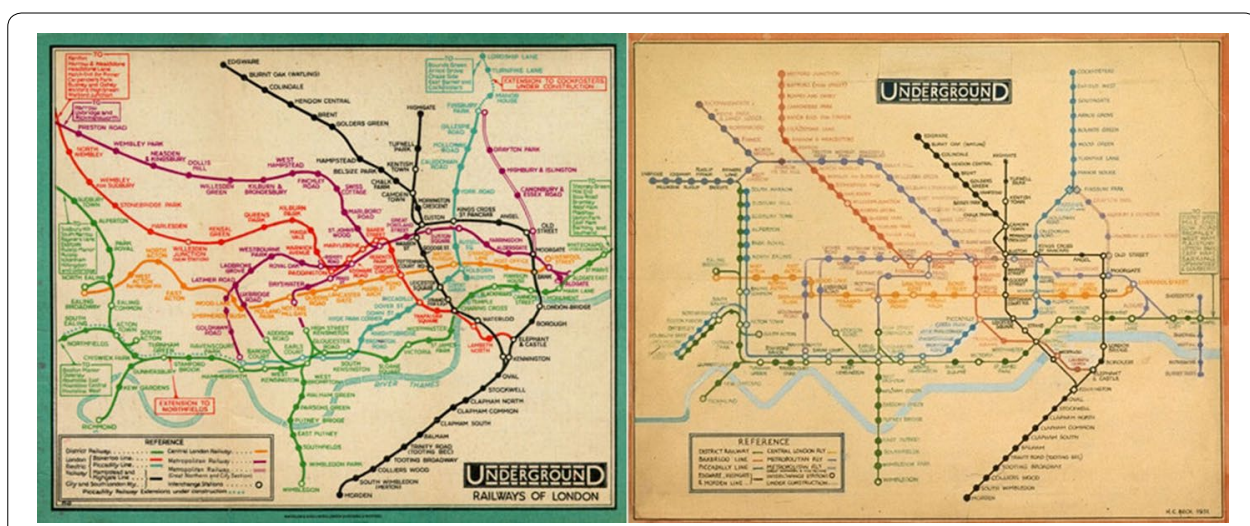

Fig. 2 Original (left) and redesign (right) map of the London underground 
Notably, the health benefits that walking brings, has led the Transport for London organization to release a "walk the tube" map (Fig. 3), in the hope that the people of London will increase the part of the routes which they do on foot [8]. Therefore, we realize that a cartographic representation of walking routes might encourage more people to move on foot, instead of motor vehicles. Besides expensive investments in improving the actual infrastructure (e.g., pedestrian streets), we suggest that one feasible way to increase the walkability of cities is to provide as much information as possible about streets that have improved walkability. For this purpose, we need to create a new kind of pedestrian map, which emphasizes the suitability of a street for walking. Indeed, it is known that the visual design of a map might influence user behavior. For example, in order to encourage the exploration of a city, the design of the Lancaster's digital map uses "dynamic visual hierarchies" [3].

The actual process of the mapmaking is based on the following pillars [4]:

- Collection of appropriate data from the field.

- Analysis and visual presentation of data according to the users' needs.

In particular, we emphasize the first pillar that regards the collection of appropriate data, because the analysis and visualization can be done easily nowadays, thanks to the availability of computing, networking, and display technology. The collection of pedestrian route data could leverage geolocation sensors widely available in contemporary mobile phones [9]. Indeed, users have voluntarily contributed information and effort in order to build geographical maps, such as openstreetmap, which are considered to be of similar quality to work performed by trained and experienced professionals [10]. Although openstreetmap has built a high quality accurate map, it has not regarded the particular needs of pedestrians, just like the rest of the digital map offerings. In most cases (open-source or commercial), pedestrian streets are highlighted on a digital map, only when they are dedicated, but, usually, streets have mixed use. Therefore, there is a

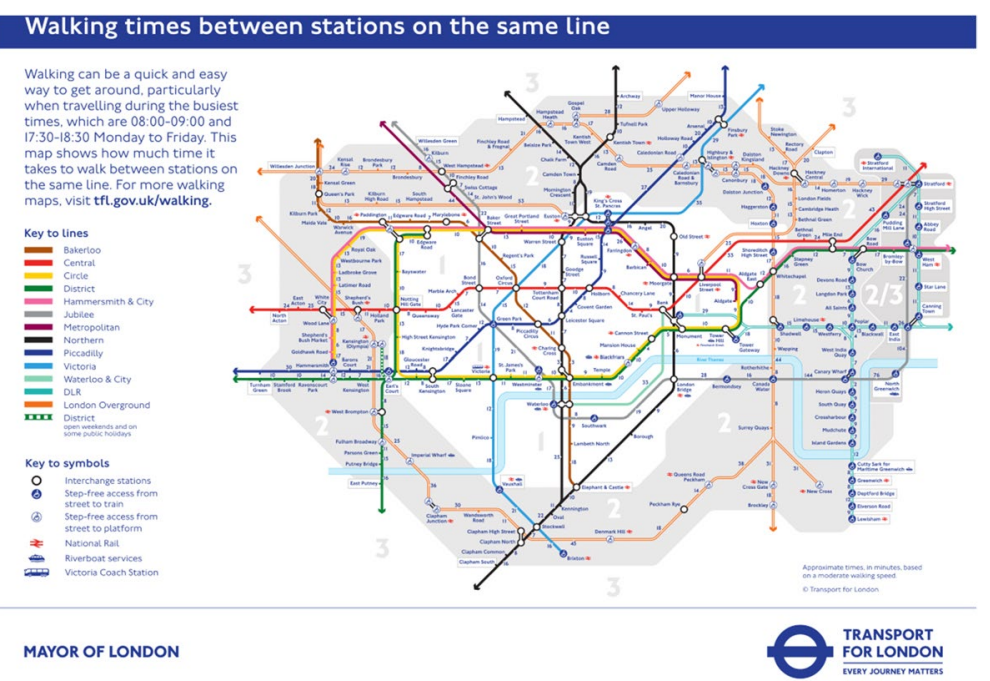

Fig. 3 'Walk the Tube' map: a map showing the walking times between stations 
need to collect data about the level of pedestrian accessibility for many streets that have mixed use (vehicle and pedestrian).

There are many efforts to improve the visibility of walkable routes, but the majority of them is focusing on existing data-sets, or only provide annotations and mash-ups of existing vehicle-oriented maps. For example, Quercia et al. [11] has leveraged the availability of Google Street View data in order to annotate and characterize streets that are suitable for pedestrians. In this way, they can annotate existing maps with pedestrianfriendly routes, but it is not known whether these routes are preferred in the actual practice of walking, because the annotation is performed on a desktop computer. In summary, previous works on mapmaking and walkability have not considered the employment of user-generated data for the transformation of a new kind of pedestrianfriendly cartography. Instead, we suggest that the creation of pedestrian-friendly cartography requires pragmatic data collected by pedestrians on the field, which will enable the production of pedestrian maps, at the next stage.

With regard to available mobile Map Apps, they are divided into two main categories: GPS recording Apps and map projection Apps. Some examples of the first category are GPS Logger for Android, OSMTracker for Android and My tracks. Their common feature is that they record tracks in GPX files. An example App from the second category is Cartographer, which displays Google maps in a beautiful user interface, which makes them look like they are old (Fig. 4-left). Furthermore, another example is the PDF Maps that reads GeoPDF maps and also allows the user to record his paths (Fig. 4-centre). Of course, most Apps with maps, nowadays, are navigators. For example, MapQuest is an App where users can ask directions on how to reach a place. It is notable that there is clear labeling of different types of roads, for example, motorways are marked with thick blue lines, main roads with yellow and one-way with arrows (Fig. 4-right).

\section{Citizen science and gamification}

Citizen science has emerged as a complementary way for collecting and analyzing data with the purpose of tackling a scientific or practical problem. According to Silvertown

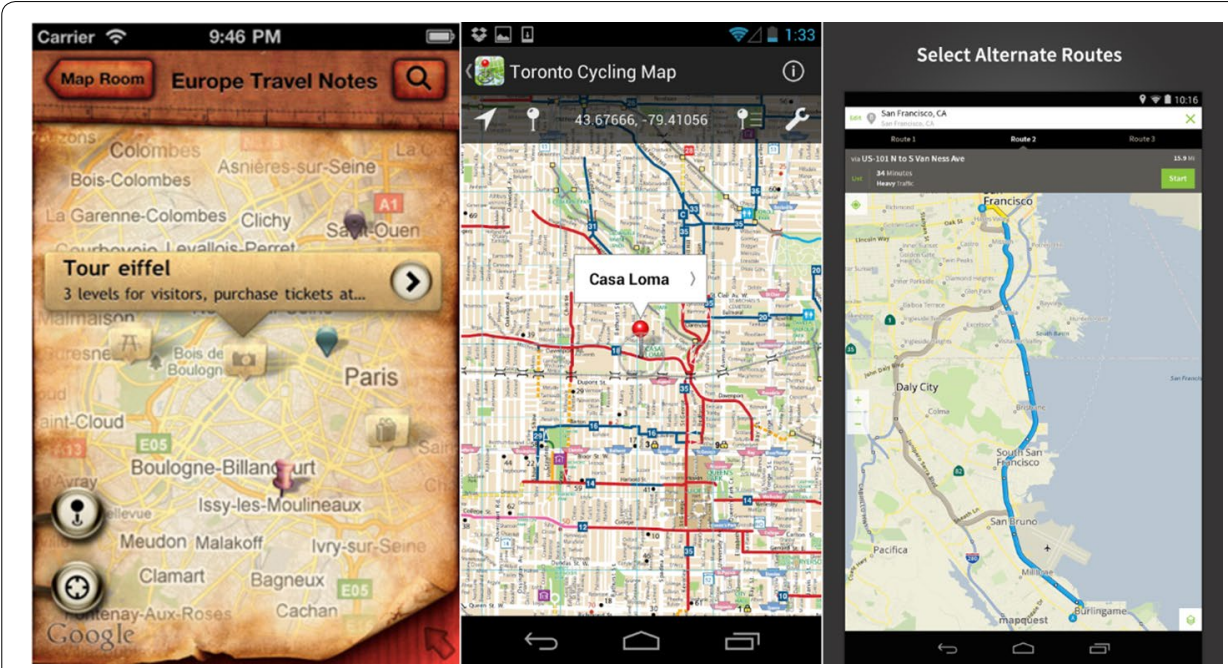

Fig. 4 Displayed map from cartographer (left), PDF maps (centre), MapQuest (right) 
[12] citizen scientist can be defined as: "A citizen scientist is a volunteer who collects and/or processes data as part of a scientific enquiry." Citizen science projects have been growing in popularity and they span scientific disciplines, especially in the life and environmental sciences. Citizens are motivated to participate in the collection and analysis of data especially when these data affect their own well-being and their quality of life [13]. Walkability in the city is naturally a suitable domain for citizen science, because the street network is very vast and complex to analyze by other means and because walkability is important for many citizens.

Besides citizen-science, participatory design has also emphasized the importance of involving users in the design, development, and improvement of systems. In particular, urban planning has embraced participatory methods for highlighting the quality of the street network with regard to pedestrians and bikers [14]. In order to create a successful crowdsourcing (or user-generated content) App, it is necessary to motivate the users to use it more often. For the design of the interaction we leverage the power of game playing. Deterding et al. [15] define "gamification" as follows: "Gamification refers to the use (rather than the extension) of design (rather than game-based technology or other game related practices) elements (rather than full-fledged games) characteristic for games (rather than play or playfulness) in non-game contexts (regardless of specific usage intentions, contexts, or media of implementation)."

Gamification has been a popular technique with many mobile Apps, which are based on user-generated content. A popular commercial (for-profit) locative media system that has successfully employed gamification is Foursquare. It started in 2009 and at the time of writing (2016), it has more than 55 million users [16]. The App encourages users to "check-in" places, by rewarding them with points and badges. The "check-in" feature has become so popular and familiar with users that has been adopted by similar applications, such as Facebook Places. In addition to "check-ins", locative media applications encourage users to provide ratings and to leave comments. It has been found that users are motivated by gamification (points, badges) to provide sensitive data (e.g., location), but few of them are also concerned about their privacy [17, 18].

Previous research has explored locative media mobile Apps and has found that they have improved the quantity and the quality of user-generated content by means of gamification. Reddy et al. [19] created a platform for cyclists where the participants could share paths and experience. This can be achieved through a mobile App that records the paths that they follow and allows them to upload geo-tagged characterized pictures or video. However, it is mentioned that users found as an inhibitory factor the phase of calibration demanded, for the recording of small paths, as well as disturbing the fact that they had to stop cycling in order to take a picture or a video. In addition, Roccetti et al. [20] created an App that suggests proper paths for disabled people. For the collection of such data they used disabled people and asked them to evaluate a path they followed for many times. In order to motivate them, they used a system of repayment, from points and ranking. Prandi et al. [21] explored ways to encourage users to upload geo-tagged photos of obstacles on their system, which identifies viable paths for people with movement difficulties. They found that gamification features increased the involvement of contributors. 
Nevertheless, an important research issue is the trust we can have on user-generated content such as ratings and check-ins. In citizen science projects, it is possible to have users that try to increase their profits through cheating or sloppiness. For example, a user might upload many irrelevant items in order to gain points, or might give negative rating to contributions by other users in order to reduce the points gained by others. For example, Eickoff et al. [22] mention that gamification can be leveraged as a technique of discouraging malicious data. Therefore, it is important to employ gamification not only for encouraging a big number of user contributions, but also for discouraging bad contributions.

\section{Mobile application design and implementation} Gamification, peer-review, and scoring system

In order to motivate the users of our App to use it as much as possible, we have been inspired by Foursquare and we employed lightweight and familiar techniques of gamification. These are the collection of points and the equivalent rating of users. Moreover, we have developed techniques for discouraging the recording of malicious data. For example, a path could have been done by car instead of walking, or a fake road tagged as pedestrian so that users can gain more points. For this reason, users are given a negative rating according to the commenting of the path from other users. The negative rating technique has been based on the ideas of Stack Overflow [23]. Thus, the negative points that a user can get if their path is commented negatively, are less than the positive in order not to discourage them from uploading a path for which they have doubts about how good it is.

The above have been implemented in a scoring system. When the user uploads a path there is a gain of 0.05 points for each meter of the path. The grading is based on the meters chosen, in order for the user to have a motive to record as many paths as possible. Also, there is a gain of 20 points for each tag that has been added along the path. The relatively many points that the user wins, motivate the user to tag the path and not to put the mobile phone in the pocket during the recording. In addition, the user gains 50 points if the path is new. The relatively many points in this case contribute to choosing new paths in order to map a city as quickly as possible.

With regard to the comments, when a path, which a user has uploaded, is commented, the user loses 30 points if the path is graded with 1 , loses 15 points if the path is graded with 2 , no points if the path is graded with 3 , the user wins 30 points if the path is graded with 4 and, the user wins 60 points if the path is graded with 5 . Furthermore, when the tags of a path uploaded by a user are commented then the user loses 30 points if the path is graded with 1 , loses 15 points if the path is graded with 2 , no points if the tags is graded with 3 , wins 40 points if the tags is graded with 4 and wins 80 points if the tags is graded with 5. Finally, each user wins 150 points for every comment of a path, since we want to test if the big amount of points is a reason why users comment a path.

\section{Geo-location and cartography}

Before choosing the right geolocation technology, we compared their theoretical capabilities. Our App has a requirement for highly accurate geolocation (e.g., within few meters), since its aim is the mapmaking for pedestrian routes, which, in some cases 
might be narrow. So, the GPS as an option of an exclusive provider seems like the best option. Nevertheless, the Global Positioning Standard (GPS) as much as the AssistedGPS (A-GPS) don't function properly in urban areas due to the limited visibility of satellites from the upper buildings [24]. This problem is significant in pedestrian routes, because they move closer to the buildings. But there are plenty of networks of Wi-Fi in a city and especially in the centre. This helps to identify the position more accurately.

Google offers the service of the fused location provider [25] for Android devices' programmers. Even though the algorithm that it uses is not public, it combines many technologies (e.g., GPS, WiFi, Cell towers) for the detection of a geo-location. Programmers can also choose if they want an increased accuracy of about $10 \mathrm{~m}$. In addition, the service promises high accuracy even inside buildings. This is something that we do want for our App when pedestrian walkways inside buildings are mapped like the Market of Chania. We compared between two alternatives for the geo-location sub-system. Kasemsuppakorn and Karimi [26] suggest an algorithm for creating a pedestrian network that comes only from GPS data from users' mobile phones. We implemented their algorithm in order to compare it to the Google fused location provider.

Paths recorded by a location sensor (ex. GPS) are not clear and need correction. For this purpose, we implemented several algorithms in the server for the correction of paths that were uploaded by users. The first filtering rejects the points that had very small accuracy, as it is suggested by Kasemsuppakorn and Karimi [26]. Next, the smoothing of the path is performed according to the algorithm of Douglas and Peucker [27]. Moreover, the Google Directions API has been used, and it enables snapping points to road [28]. This was carried out only for the tagging of the roads since there are API quotas that prevent its use for the entire length of the path. Post-processing of geolocation data is quite common in mapmaking. For example Google Maps are created by a combination of algorithms and elaborate manual effort [29]. Indeed, Google uses a small army of operators to align the paths from the algorithms with the paths of the satellite image, by dragging them (Fig. 5). Therefore, it is impossible to optimize any map without significant manual effort.

We wanted to support base layer maps in our App in order to allow the removal of the existing layer of the road-network, which should be replaced by the pedestrians' layer. This is possible in the Google Maps API v3, which was used not only for the illustration of the pedestrians' maps but also for the illustration of other users' paths. We also used the Google Maps API v2 because it is free and does not have restrictions in order to reduce the possibility of service interruption.

\section{System architecture}

The mobile App was created for the Android operating system. In the fourth term of 2015 the Android was leading in the market of mobile phones with an 80.7\% [30]. For the server, we employed established technologies such as PHP and MySQL that were satisfying for our App. In addition, for some operations the language JavaScript has been used, such as the illustration of users' paths on the server.

The system is based on the client-server architecture (Fig. 6). The server implements an application programming interface (API) that is responsible for serving queries made by the client as well as for data storage such as storage paths of users in GPX files or 


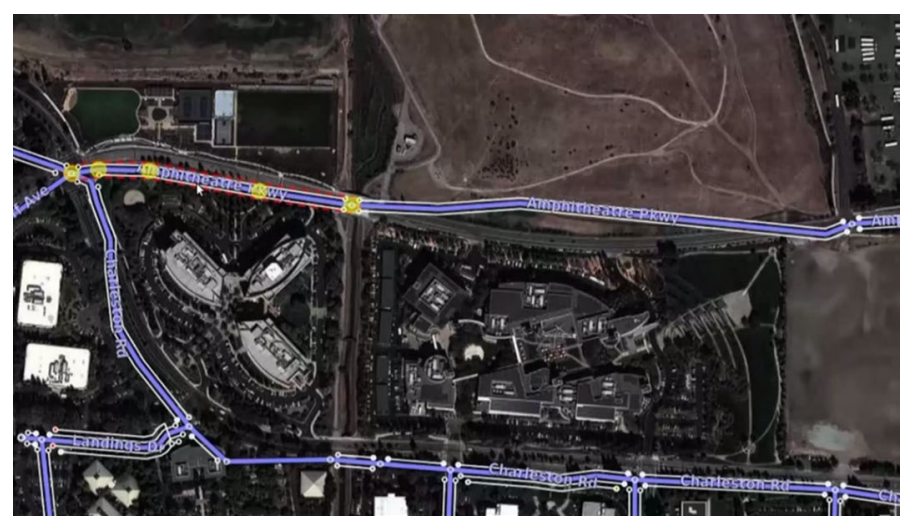

Fig. 5 Operators can highlight a road and drag it into alignment with satellite imagery

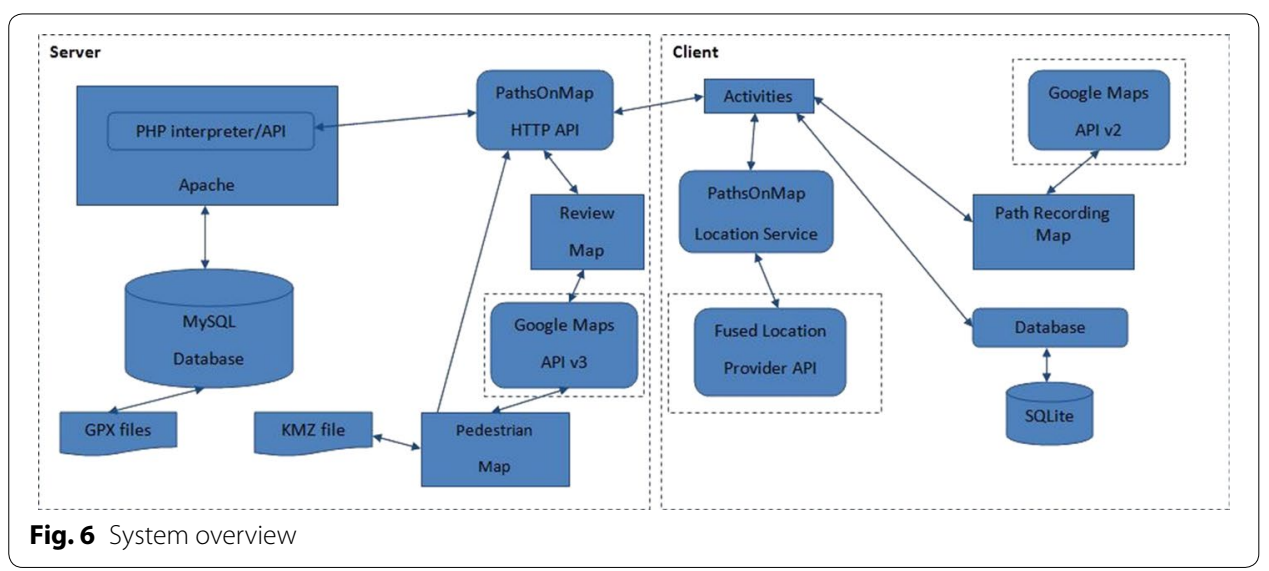

users' reviews, by means of a MySQL Database. Also, it is responsible for sending to the client a review or pedestrian map. On the client, there are activities that get (or send) data from the server, using HTTP queries. Moreover, there is a SQLite database that stores user data, and which is managed by the Database Handler. In addition, on the client is a Location Service, which is responsible for recording the user's path. Finally, it is worth noting that the Path Recording Map is created within the client.

The recording of users' paths has been done in GPX files which are the most widespread files for exchanging geographical data and in this way the system might provide linkeddata to other systems. Google Maps API v3 has been chosen for the illustration of the final pedestrians' map. It allows only the KML level (and not GPX) for the illustration of the pedestrians' road level in a city, for this reason a KMZ file (zipped KML file) has been used.

\section{User interface}

The main screen of the App shows the map of a city of interest with the paths that have been recorded by the users until now with a red color (Fig. 7-left). The user is given the ability to record a path that they will follow by pressing the button "Start Path". If this happens, the App finds the position of the user and if this is located in the town of interest it starts recording of the path. 


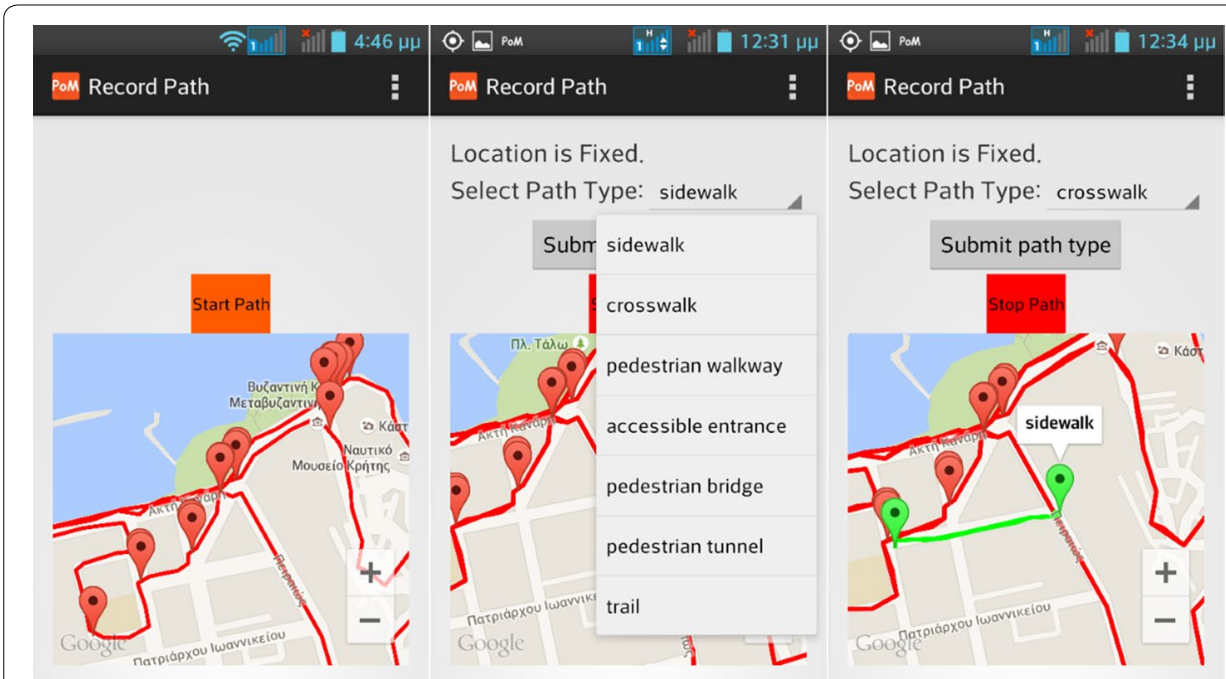

Fig. 7 Paths of other users (left), choices of types of maps (centre), recording of the user's path (right)

The user's path is recorded with a green color so as to be distinguished by the other paths (Fig. 7-right). At the same time, there is also a spinner so the user is able to choose the type of path they are in every moment (Fig. 7-centre). The user must make this choice every time they change types of path. Here is the range of choices: (1) sidewalk, (2) crosswalk, (3) pedestrian walkway, (4) accessible entrance, (5) pedestrian bridge, (6) pedestrian tunnel, (7) trail. We chose this distinction between seven types of paths for pedestrians, according to the Kasemsuppakorn and Karimi [31] project.

When the user reaches the end of their path, the App transports him to another screen that encourages him to upload the path and informs the user about the points that he will win if they do it (Fig. 8-left). Moreover, at any moment the user is able to go to the ranking screen (Fig. 8-centre). In addition, the user can also visit the peer-review screen in order to evaluate another user's path (Fig. 8-right). In the review screen the user can choose to see the tagging data (Fig. 9-left). The review is made for the path (Fig. 9-centre) and separately for the tagging of the path. Finally, the user can choose to see the pedestrians' map that has been created until now (Fig. 9-right).

\section{Exploratory study}

Before the final evaluation, we performed an exploratory study, in order to improve the usability of the mobile App and to test the robustness of the server side. For the exploratory evaluation, we first created one App for Chania. Besides the behavioral data of walkable routes and peer-reviews of uploaded routes, we employed unstructured interviews, in order to gain in-depth understanding of users' motivations and attitudes. Five users were recruited (Table 1) and they were asked to use the App for 2 weeks with the goal of competing for more points.

The conclusions extracted from the interview were:

- The gamification (points and rating) motivates users to record more paths in order to compete with others on the leaderboard. 


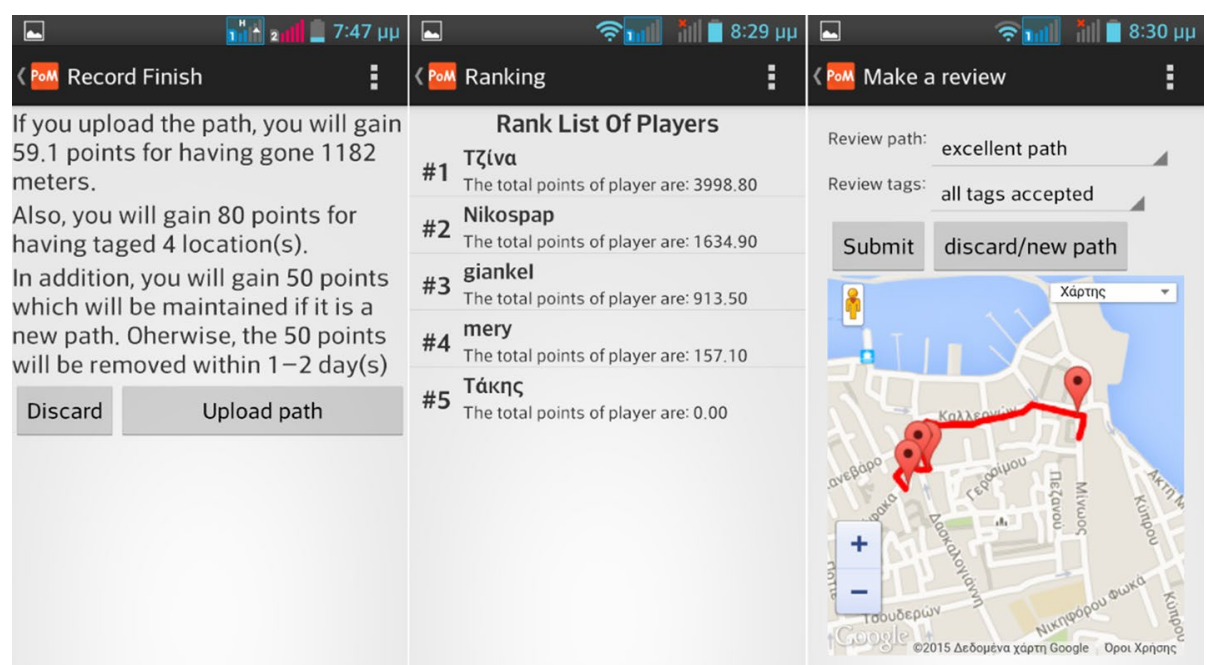

Fig. 8 Screen for the uploading of a path (left), ranking of users (centre), review screen (right)

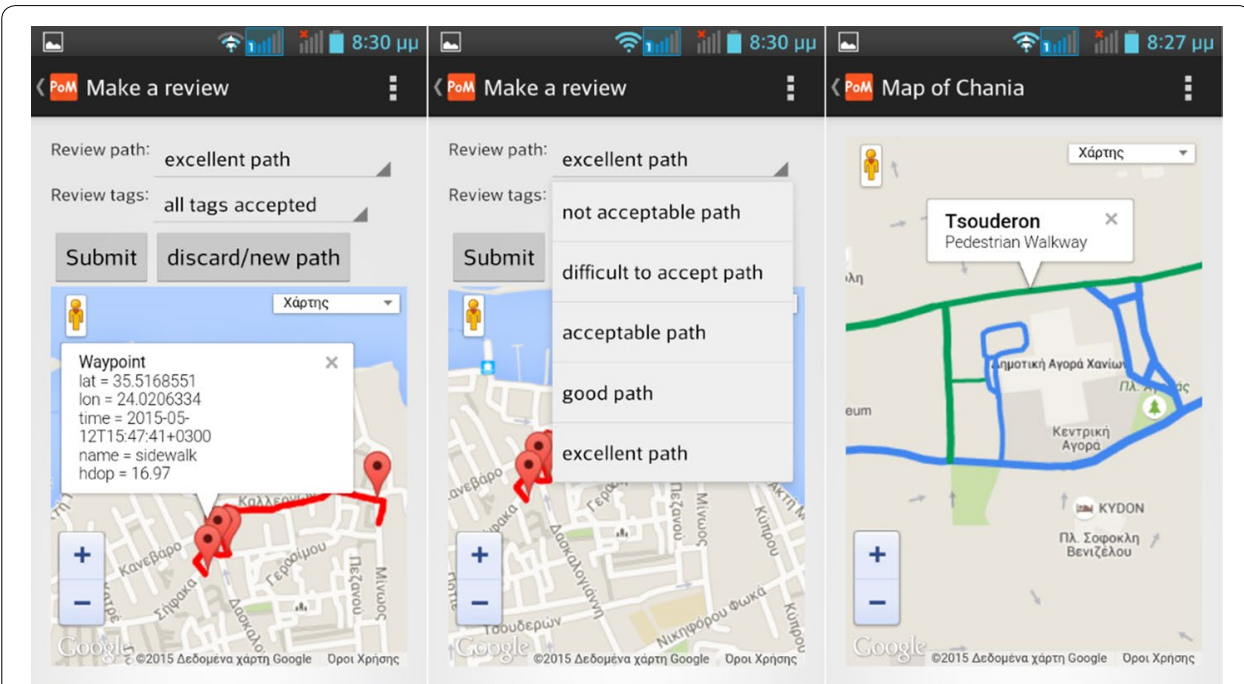

Fig. 9 Tagging data (left), options for commenting a path (centre), pedestrians' map with road information (right)

Table 1 Table of users

\begin{tabular}{llllll}
\hline Users & Genre & Age & Town of living Chania & $\begin{array}{l}\text { Foot as a usual means } \\
\text { of transport }\end{array}$ & $\begin{array}{l}\text { Previous experience } \\
\text { of recording applications }\end{array}$ \\
\hline User 1 & Female & 32 & Yes & Yes & No \\
User 2 & Male & 38 & Yes & Yes & Yes \\
User 3 & Female & 40 & Yes & Yes & No \\
User 4 & Male & 36 & Yes & Yes & No \\
User 5 & Male & 30 & Yes & Yes & Yes \\
\hline
\end{tabular}


- The map choice that demonstrates paths of other users during the recording motivates users to record new paths.

- The demand for tagging routes was considered as annoying.

\section{Results}

\section{Participants, method, and attitudes}

In the final stage, we did a longitudinal evaluation in order to address the main research question, by carrying out a quantitative evaluation. For this purpose, we created an App for Corfu (Greece). The App was given to 13 students at the Department of Informatics in the Ionian University and asked to use it for 7 weeks. The majority of users was male (84.6\%) and 19-25 years old (92.3\%). All used their feet as a means of transport and the majority was familiar with an Android mobile phone, since $76.9 \%$ of the users had one in their possession for over a year. Also, 61.5\% of the users had used a GPS recording App (Fig. 10). As a reward, the students would receive a bonus grade in a course on HumanComputer Interaction. It should be noted that the App included Google Analytics for measuring users' engagement. Afterwards, users were asked to complete a questionnaire on their experience of using the App. Finally, 9 out of 13 users (Table 2), who downloaded the App, uploaded paths to the server.

We extracted several interesting conclusions from the examination of users' attitudes:

First of all, the recording of a path did not seem to make it difficult for a user, since everyone felt that the process was easy to some extent (Fig. 11).

Also, the average length of paths was about $1300 \mathrm{~m}$ and the average length of path, held by the subject who made the smaller paths, was $522 \mathrm{~m}$. Therefore, the 0.05 points gained by players for each meter of path are satisfactory for failing to take short paths, just to gain 50 points which be gained by a new path. Indeed, the gain of points seems to have affected a $23.1 \%$ perform longer paths, as shown by their answers (Fig. 11).

Furthermore, the 90 paths performed during the experiment, appear sufficient to create a relatively complete pedestrian map. This is also because, as shown by the answers of the users, the majority of them, which was a $53.9 \%(38.5+15.4 \%)$, influenced by the routes of other users and performing a new path to gain more points (Fig. 11). Also, the design of the App helped, as revealed by the interview of the previous experiment, since $100 \%$ of users understood the significance of the red and green lines. Finally, it is worth noting that the gain of points influenced a $30.8 \%(23.1+7.7 \%)$ to perform more routes (Fig. 11).

It is worth noting that six of the nine participants who uploaded paths, put on average one or fewer tags per path, although they changed road type. Indeed, from the answers of the users, it seems that a $38.5 \%(23.1+15.4 \%)$ influenced little or not at all, and another $38.5 \%$ was not influenced as much as we hoped, by the gain of points for this purpose (Fig. 12). Also, a $61.5 \%(53.8+7.7 \%)$ considered annoying factor having to hold the mobile phone during the path for this purpose (Fig. 12). This is in agreement with the conclusion of the interview in the previous experiment. Indeed, the nontagging of the paths, it should not be seen as a lack of understanding of tags use, since $53.9 \%(38.5+15.4 \%)$ stated that they understood their use much to very much and an additional $30.8 \%$ said they understood enough (Fig. 12). 


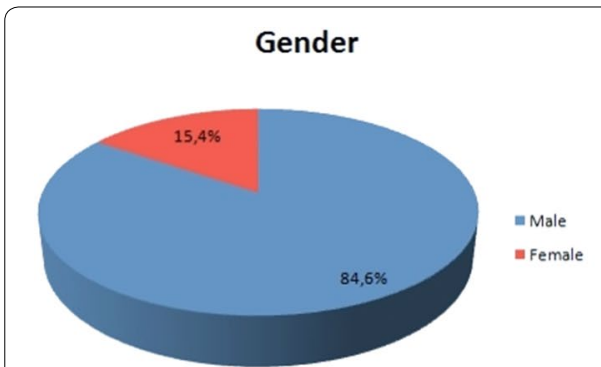

Are you often using your feet as means of transport?

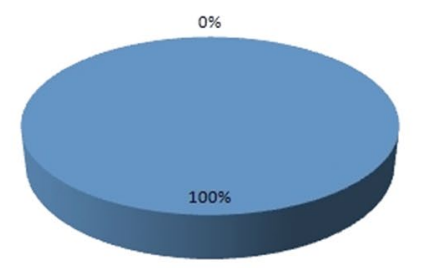

- Yes

No

Ires

ano

Have you had any previous experience of GPS recording applications?

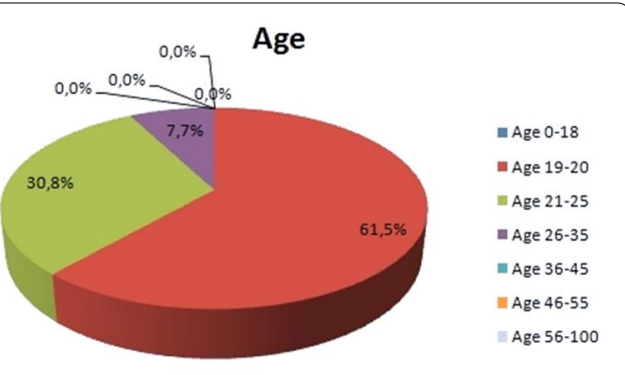

How long do you have a mobile phone with Android operating system?

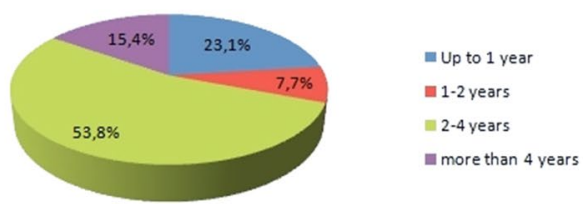

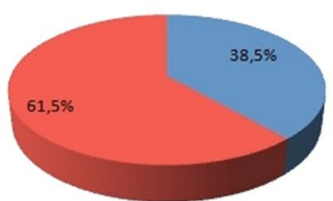

Fig. 10 The subjects' information

Table 2 The participant information taken by each subject as recorded on the server

\begin{tabular}{lclclcc}
\hline Users & $\begin{array}{l}\text { Total distance } \\
\text { traveled } \\
\text { in meters }\end{array}$ & $\begin{array}{l}\text { Average route } \\
\text { distance } \\
\text { in meters }\end{array}$ & Total tags & $\begin{array}{l}\text { Average tags } \\
\text { per path }\end{array}$ & $\begin{array}{l}\text { Number } \\
\text { of routes }\end{array}$ & $\begin{array}{l}\text { Number } \\
\text { of reviews }\end{array}$ \\
\hline Subject 1 & 0 & 0 & 0 & 0 & 0 & 0 \\
Subject 2 & 3789 & 541.2857 & 26 & 3.7 & 7 & 0 \\
Subject 3 & 1439 & 719.5000 & 2 & 1 & 2 & 0 \\
Subject 4 & 22,954 & 1093.0476 & 19 & 0.9 & 21 & 1 \\
Subject 5 & 32,680 & 1021.25 & 2 & 0.06 & 32 & 68 \\
Subject 6 & 5454 & 1818.00 & 3 & 1 & 3 & 0 \\
Subject 7 & 0 & 0 & 0 & 0 & 0 & 0 \\
Subject 8 & 14,027 & 1402.70 & 1 & 0.10 & 10 & 76 \\
Subject 9 & 28,601 & 2860.10 & 127 & 12.7 & 10 & 69 \\
Subject 10 & 522 & 522.00 & 1 & 1 & 1 & 0 \\
Subject 11 & 0 & 0 & 0 & 0 & 0 & 0 \\
Subject 12 & 0 & 0 & 0 & 0 & 0 & 0 \\
Subject 13 & 5507 & 1376.7500 & 5 & 1.25 & 4 & 0 \\
\hline
\end{tabular}




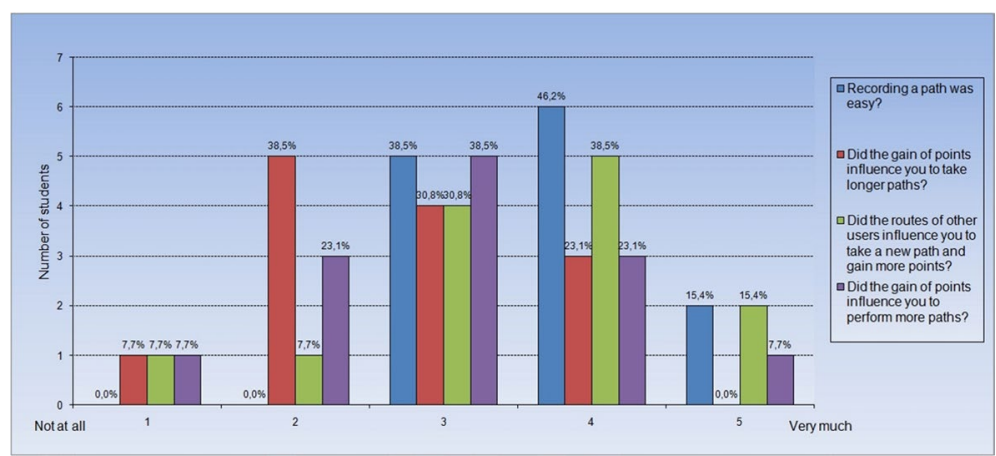

Fig. 11 The answers of the subjects (first questions)

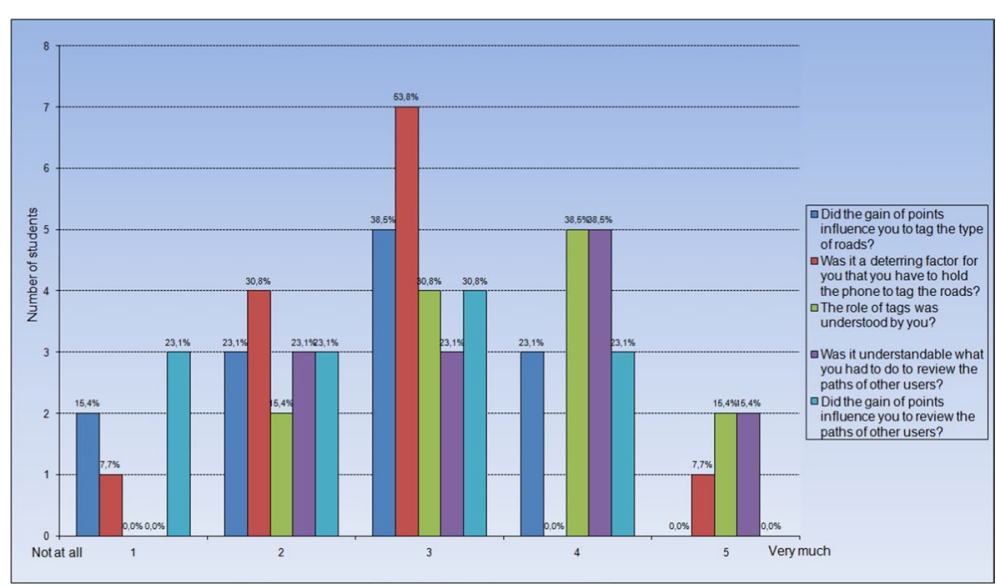

Fig. 12 The answers of the subjects (second questions)

Last but not least, there were seven users (53.9\%) who understood much or very much what to do for review the paths of other users (Fig. 12). We believe that this percentage is relatively small due to the fact that no explanations were given to users and also the App did not have any help. Of these, three users (23.1\%) were influenced by the gain of points in order to review the paths of other users (Fig. 12). In fact, as shown in Table 2, only four users tried to review the paths of other users. Therefore, we believe that the points they were gaining, was an important factor in order to review on a large number of paths. Unfortunately, as shown in Fig. 13, only one of the three users (with uid 10), appears to be rated according to the reality the paths of other users. The second subject (with uid 14) seems to have rated lower the paths of other users, at least in beginning, possible in order other players to lose points, so he can get ahead in score. The third subject (with uid 13) seems that he has rated with "excellent" the paths of all users, in order just to win points easily. While the results show that the points are important to encourage players to review on the paths of other users, however, we need to find a more effective way of scoring for this purpose, e.g. loss of points for users who comment negatively good paths of other users. 


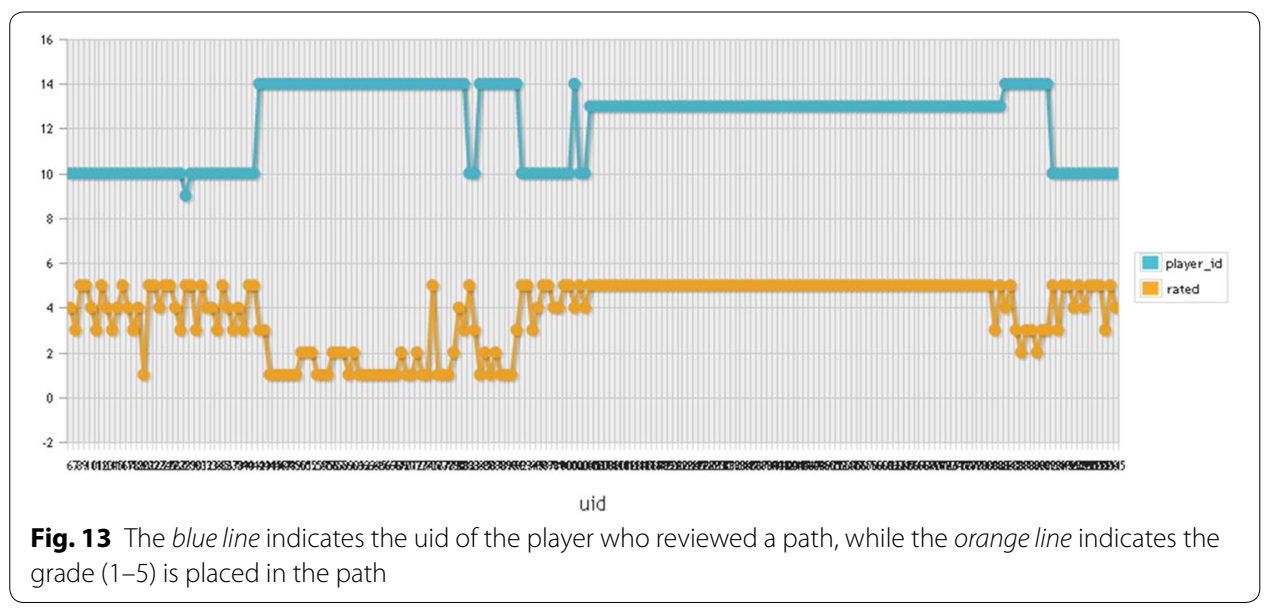

\section{Users' engagement and privacy}

The majority of users didn't lose their interest during the experimental period, since the number of users who used the App was almost constant during the experiment, like Google Analytics showed (Fig. 14). We believe this is due, to some extent, to gamification of the App, since we noticed some users were trying to keep or increase their ranking.

It is worth mentioning that during the recruitment of participants, we noticed that the invasion of privacy is an inhibitory factor for mobile Apps that record paths. We protect user privacy because the App doesn't record all the time. The user can choose which path is going to be recorded and if it should be uploaded at the system for post-processing and inclusion in the aggregate pedestrian cartography. Moreover, uploaded paths are shown to other users for evaluation, but they can't see who uploaded them. However, we met potentials participants that answered they wouldn't upload their paths, for any reason. This is normal because there will always be a group of users-about $25-30 \%$ according to Kumaraguru and Cranor [32] - that are reluctant to participate in any type of user-generated content App.

\section{Comparison of GPS and fused location provider}

During the field study, we gathered very useful data on the data base of the server, from which we can compare GPS and fused location provider.

Fused location provider in a combination with the smoothing algorithm is the best choice. This can be seen in Fig. 15 in which has been recorded a path from fused location

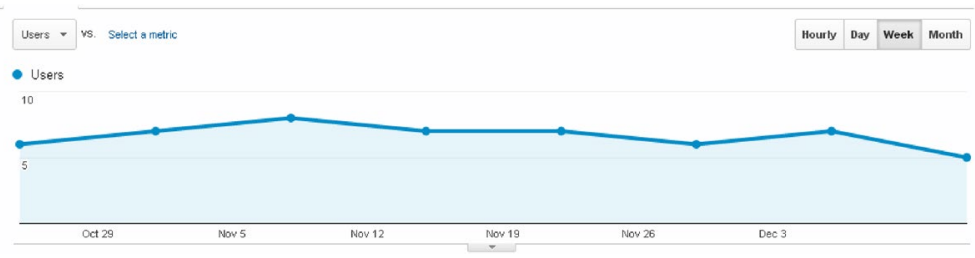

Fig. 14 Number of users who used the application per week 


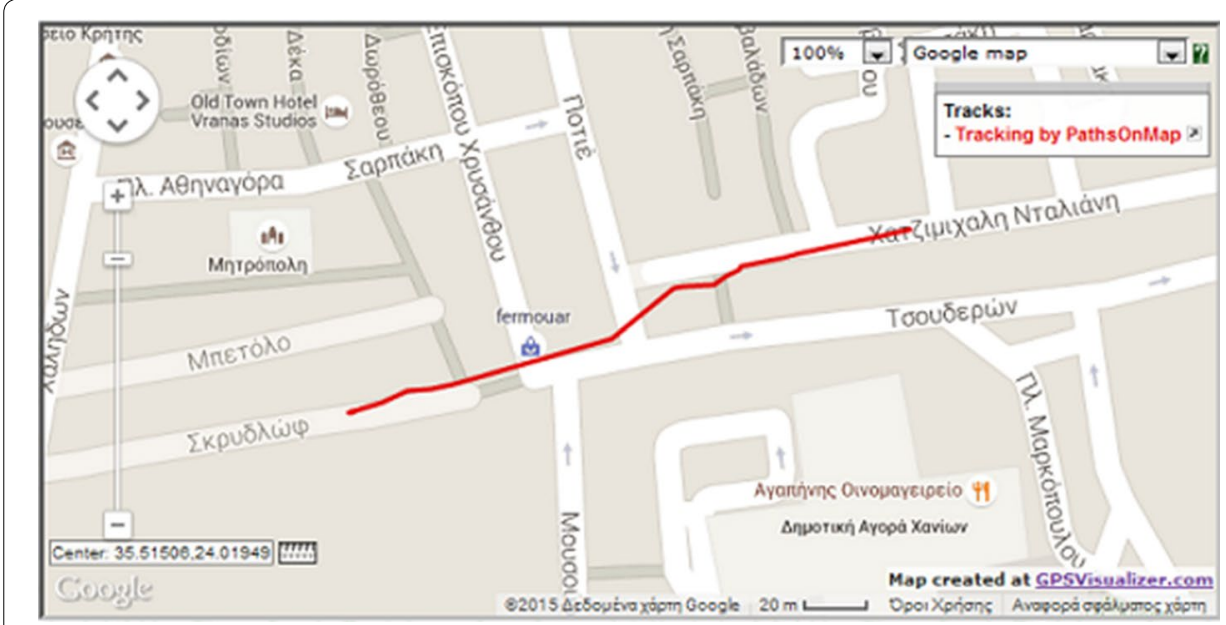

Fig. 15 Recorded map with fused location provider

provider and for which the GPS didn't give any position, as well as in Fig. 16 in which there is a comparison of a path with just the use of GPS provider, just the use of fused location provider and in the end, the fused location provider combined with the use of smoothing algorithm and of "snap to road" for tagging. The "snap to road" works very well for tagging (Fig. 16).

\section{Mapmaking}

The next step after the data collection of walkable routes is to represent these data in cartographic format. In this section we briefly describe the way we created the pedestrian map in order to assess whether its creation is possible from user-generated data. Firstly, we developed an administrator's website, which displays on a digital map (Fig. 17) one by one all the routes that have been uploaded to the server by the users. Next, we used the My Maps application from Google, and for each path that was uploaded, and which was rated positively or was not rated, we were creating the corresponding line (Fig. 18). If a piece of the path has already been uploaded, then we were increasing the thickness of the corresponding line. Moreover, for each new street we assigned a color according to the tags that were put by users. For example, if they had put one sidewalk tag, then we colored the line blue (Figs. 17, 18). Then, once we have created the map, we

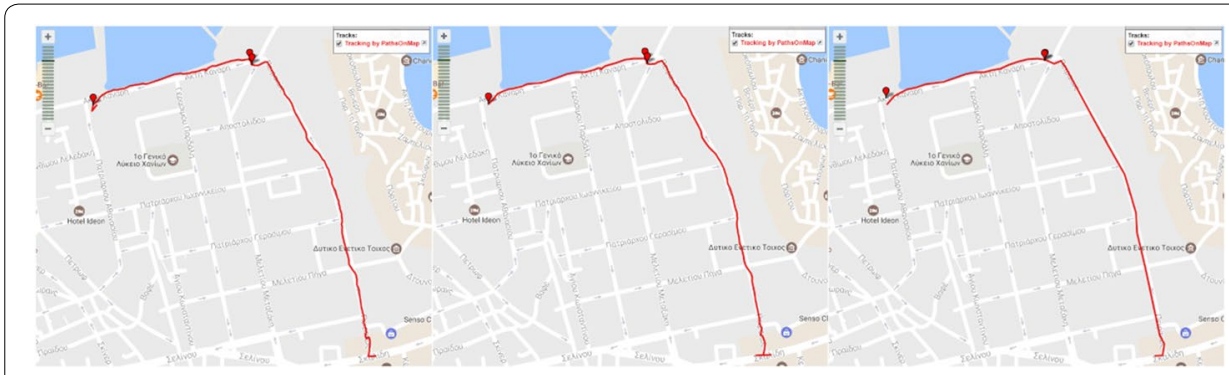

Fig. 16 Recording of the path with the use: (1) of the GPS provider only (left), (2) of the fused location provider only (centre), (3) of the fused location provider combined with the smoothing algorithm and the "snap to road"for tagging (right) 


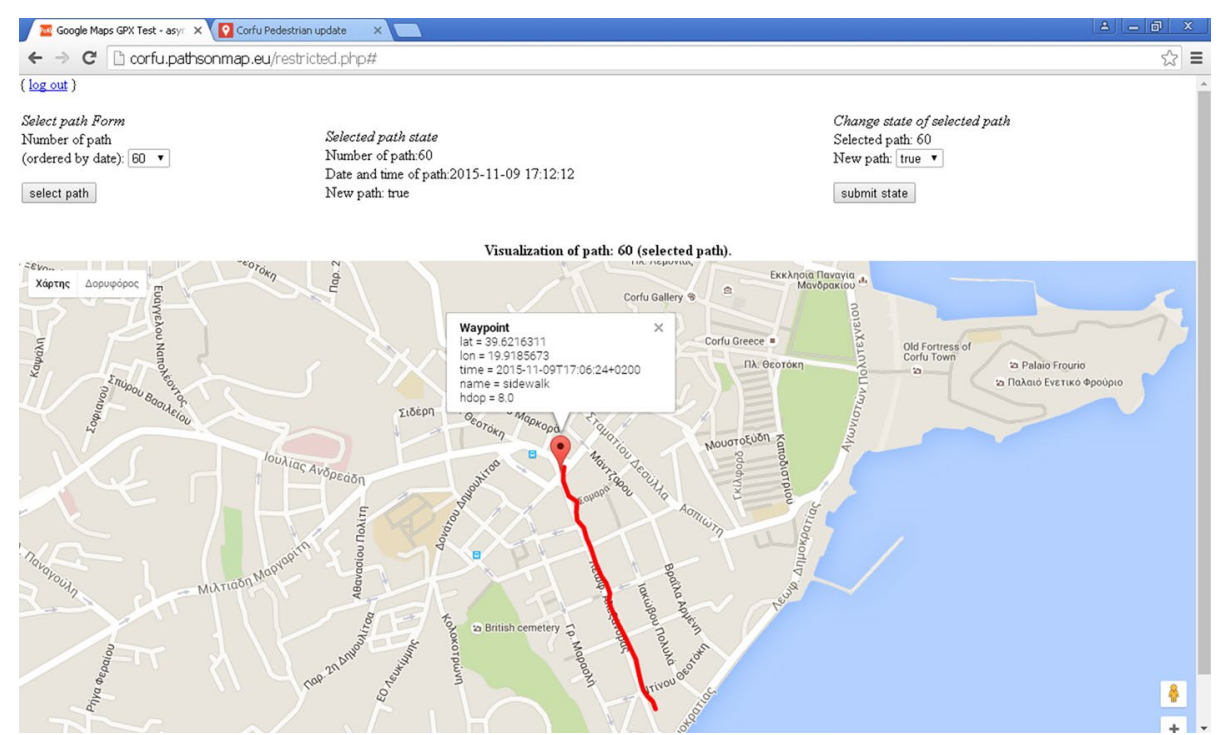

Fig. 17 Administrator's website

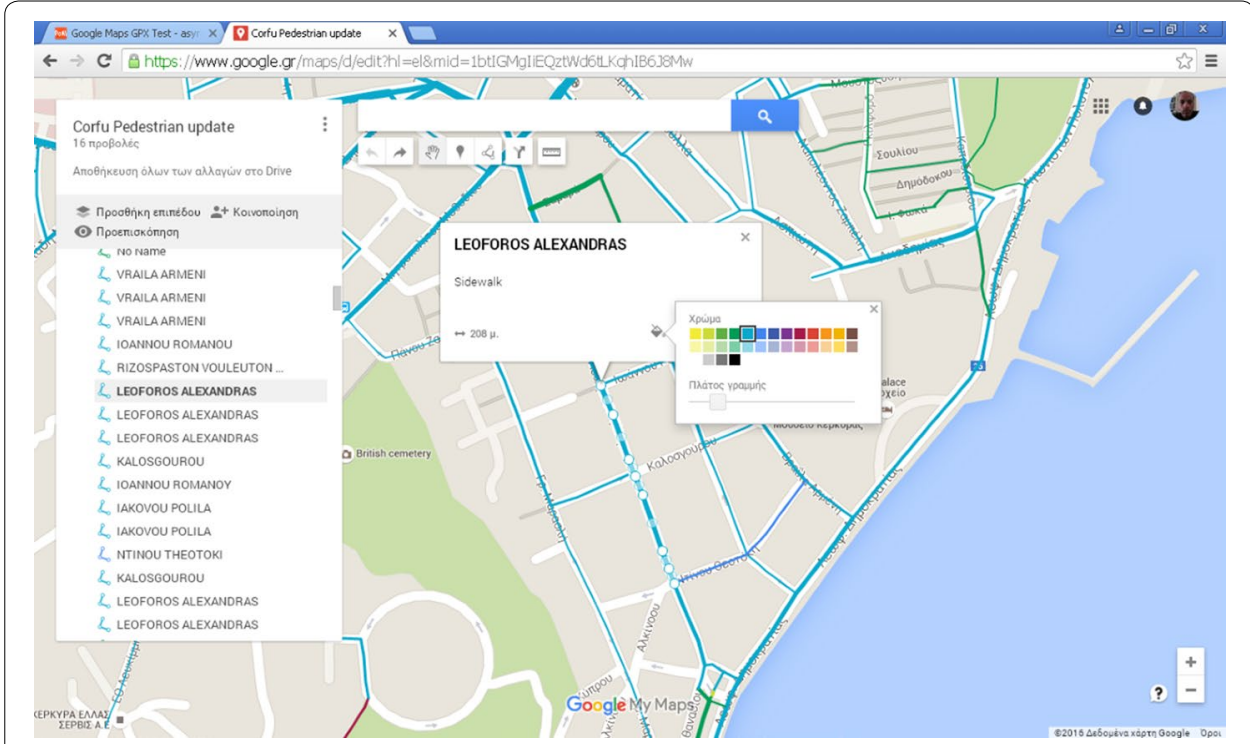

Fig. 18 Creation of map with the help of my maps application

saved it as a KMZ file and we uploaded it to the server so it can be displayed from our application. Moreover, we have created a heatmap by automatically merging the paths (without their type), the result of which is shown in Fig. 19. Ideally, we would like to have as much as possible automatic creation of the map from the user-generated routes, but this is a subject of future work, as discussed in the next section.

\section{Discussion}

\section{Pedestrian mapmaking and mobile App system}

The task of mapmaking requires data collection and produces the visual format of cartography, which can be regarded as a user interface for navigating the physical world. 


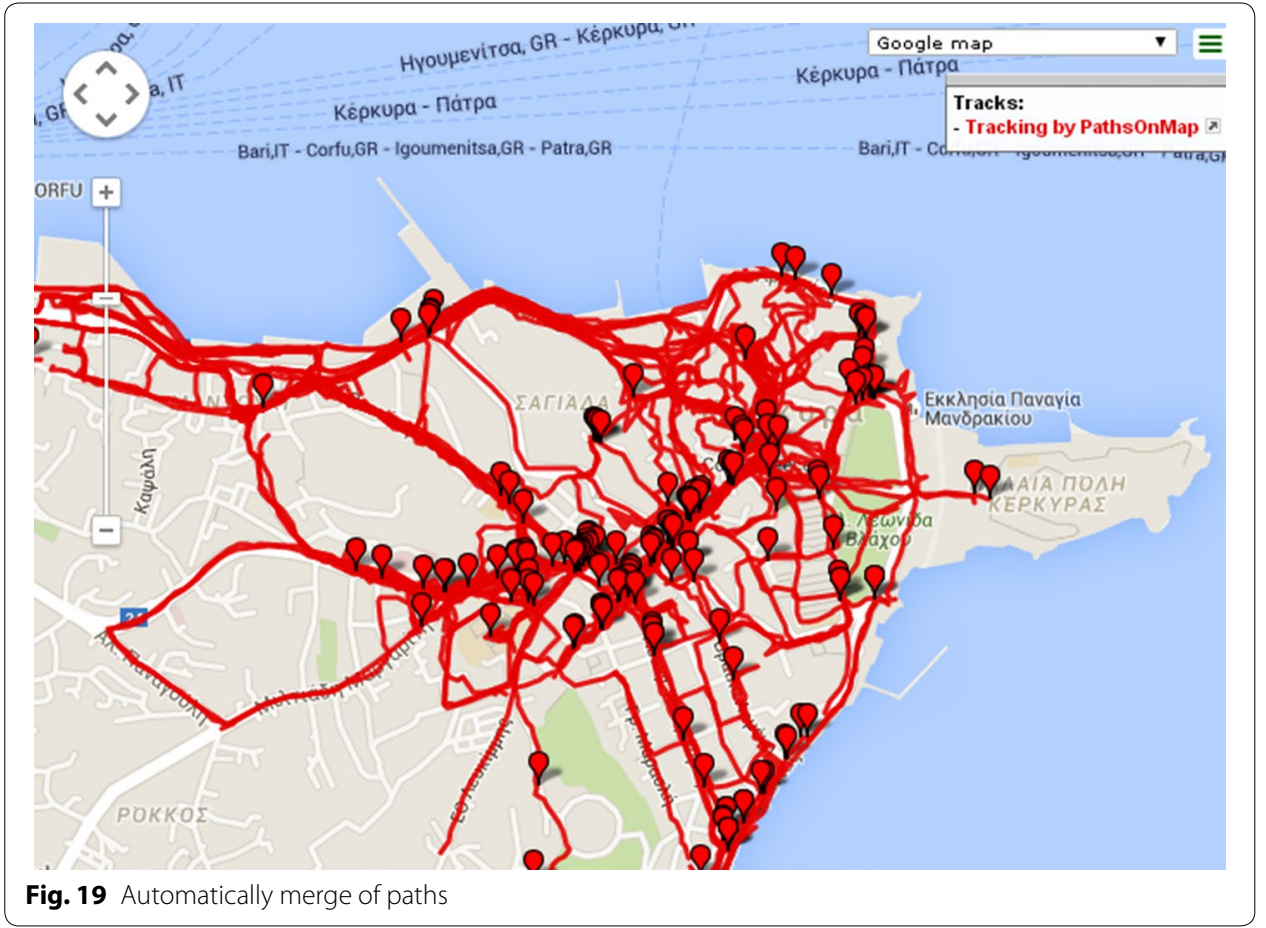

The most important activity in mapmaking is the collection of suitable data. We suggest that in the case of pedestrians the data consist of actual routes traveled on foot. A mobile App enables the users to record and upload a route. Due to the inaccuracy of the GPS sensor in a city environment, we used Google's fused location provider, which can give a highly precision in urban areas. Indeed, the time to first fix is reduced and we believe that this can improve recording of pedestrian routes. For example, users won't be discouraged to record short paths, such as the Biketastic [19] that demands users to spend some time for the calibration of the App.

In this work, we have delegated the collection of data to users through crowdsourcing, but, in the current version, there is also a need for manual effort in order to visualize the data into a useful cartography. We do not expect that a pedestrian cartography could be complete without some manual effort during the post-processing of uploaded routes. Indeed, manual effort is demanded for the creation of cartography, which is something that Google also does for its popular map system [29]. We suggest that an important challenge for the future is to increase the automation in the creation of a pedestrian map, but we expect that there should also be some manual effort. Although some features did not work-out as expected (e.g., tags), the recording of routes with minor post-processing has resulted in effective pedestrian cartography (Fig. 20) that is significantly different to a typical digital map, which emphasizes (implicitly) motor vehicles (Fig. 21).

Previous works in pedestrian navigation have highlighted the importance (and the lack of) pedestrian-centred navigation, but they have not produced new cartographies or open systems. Traunmueller et al. [33] have created a pedestrian navigation mobile App (Likeways iOS App) that leverages social media check-ins (e.g., Facebook places) in order to infer social places, which become waypoints in a suggested route. Nevertheless, the Likeways App makes the implicit assumption that users want only to go 


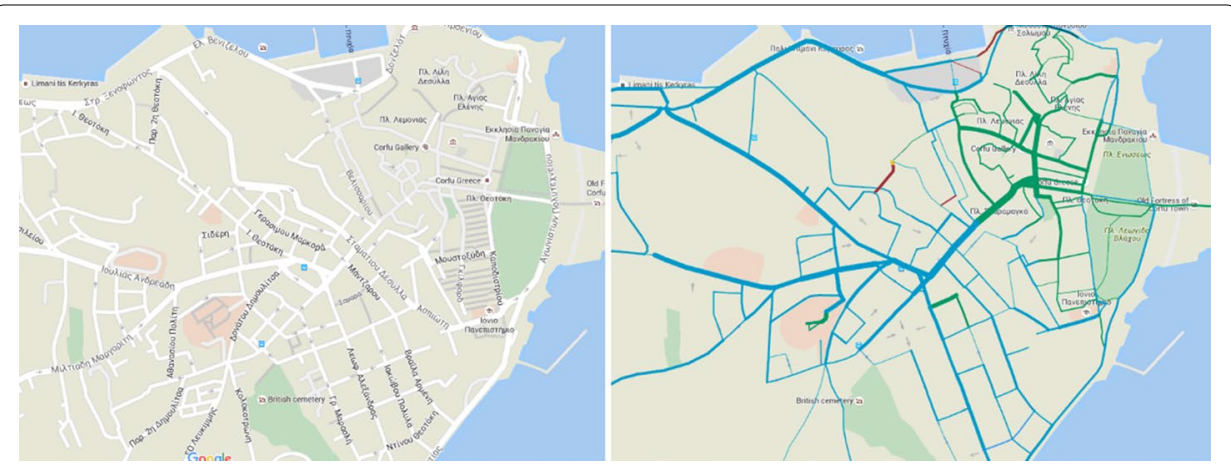

Fig. 20 The comparison between the original Google Map (left) and the one that is based on actual walkable routes travelled by city dwellers (right) demonstrates the differences in perspective between a motor- and pedestrian-centred cartography

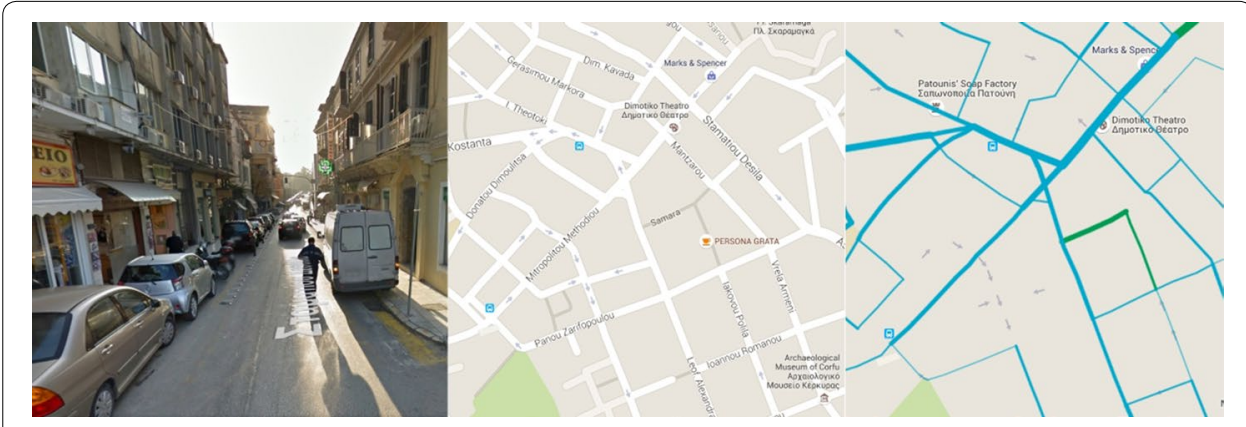

Fig. 21 In addition to the streets mentioned in the introduction, Stamatiou Desila street is visualized with a rather wide line at the top right of the Google Map (centre), but it has not been preferred by pedestrians, since it is a noise street, busy with motor cars, and small pavements (left). Stamatiou Desial street, which is close to Marks and Spencer, is visualized with a rather thin line at the top right of the pedestrian map (right), because it is not considered as a walkable street

through social routes. The City 'R' Us App (Android) asks users to upload routes according to means of transport (e.g., walking, running, biking, accessibility, etc.), which are displayed on a standard (motor vehicle-centered) digital map. Among the many social Apps, there is also a contrarian App created by Posti et al. [34], which promotes asocial hiking. The App suggests isolated paths, which are less likely to have other people based on geotagged photos from Flickr and wifi-sniffing. This App suggests that sometimes a pedestrian might opt for an isolated route, thus the sociability of a pedestrian map layer should probably be just a parameter among many others, such as accessibility, safety, nature, sightseeings, shops, etc. The above are useful mobile Apps, but their goals and utility does not extend beyond the App itself. In contrast, our open-source App and the back-end system are just a means to an end, because they employ gamification and crowdsourcing in order to create a new kind of cartography.

In summary, we have developed and tested in the field a mobile App and the corresponding back-end service that facilitate the collection and processing of pedestrian routes in a semi-automatic way. Previous works have also employed a participatory approach in the data collection [14], but they have regarded neither automatic geo-location nor engagement through gamification. In our work, the majority of the workload 
has been delegated to end-users through a crowdsourcing of their actual routes, which is based on gamification. We have confirmed Haklay et al. [35] that even a small number of motivated users who performed a few kilometers each $(2-5 \mathrm{~km})$ has produced enough data to create a useful pedestrian map for a small city of forty thousand inhabitants. In addition to demonstrating the feasibility of this App in the field, we have made the source code of the server and the mobile App available for other researchers, in order to validate, improve it, and hopefully apply it to similar scenarios (e.g., biking, accessibility) that require the collection and analysis of routes. In the current field study, we aimed mainly to evaluate the feasibility of the idea and of the system, so the analysis of the results are rather qualitative than quantitative, because there are only a few participants. Further research, should consider validating the system for larger cities and with more participants, which should allow tests of statistical significance, too.

\section{Citizen-science and gamification}

In addition to validating a mobile App system that facilitates the collection and processing of route data, we have employed gamification elements as a means to motivate users to participate in a common effort for mutual benefit. In particular, we employed a simple scoring system for route collection as well as for peer-review of collected routes. We employed gamification similarly to Quercia et al. [11] and Roccetti et al. [20] and we found that more than a few users have been highly motivated to find new routes and to moderate bad behavior. In contrast to Roccetti et al. [20], we gave the users the ability to upload whatever route they want, even if they don't usually follow it, because in our research we aim to record all possible pedestrian routes. Although the gamification of the mobile application could be considered as lightweight in comparison to related work (e.g., [21]), it has been sufficient to collect enough data for preparing a pedestrian map of a small city with forty thousand inhabitants. Moreover, similar lightweight gamification methods have proven very successful in commercial services, such Foursquare, which motivates user to check-in just by offering (mayor) badges. Overall, we found that even a simple point system that encourages route uploading and moderation is enough to bootstrap pedestrian cartography that is significantly better than popular digital maps.

Although gamification offers a wide spectrum of game elements, we found that only some of them have been useful in our App. The type of route followed by pedestrians could be potentially useful in mapmaking, so we created a user interface menu in order to be able to choose easily the type, instead of asking them to take pictures or videos that had been characterized as annoying in the work of Redy et al. [19]. Motivating users to add road tags through generous points, hasn't been effective enough, since there were many users that didn't add tags. A possible ergonomic explanation is that users want to have their hands free during their walk. In further research, we suggest the expansion of the App on smart watches of users. Nevertheless, there are some concerns about privacy and geo-location with many users, who are not comfortable to participate at all.

Gamification has provided significant motivation for many users to upload and to peer-review routes, but it has also created motivation for cheating. Few users have tried to give negative or low scores to excellent contributions. There are two possible solutions, one is to update the scoring system and the second is to have an additional user group with super-user privileges. This kind of behavior is common in citizen-science 
and user generated content projects. We have been able to monitor and report this kind behavior because we deliberately employed a small number of users in a small city that we are familiar with. Nevertheless, in order to scale this system to more users and cities, there is a need for additional administrative user's interfaces that facilitate monitoring. Further practical systems should consider to have expert and trustworthy committees of users that monitor and ban bad behavior, just like Wikipedia. Therefore, we suggest that pragmatic pedestrian cartography could be deployed initially in urban workshops with few dedicated users, in order to create a strong and trustworthy community that is going to monitor the system as it expands to more users. In further research, we suggest a comparative study with different types of users (e.g., urban planning students, citizens), in order to be able to draw conclusions about the type of motivations (intrinsic or extrinsic).

In summary, we found that citizen science through mobile App gamification is a feasible means for pedestrian mapmaking, just like it has been for popular locative media. The gamification user interface that we employed is conceptually similar and inspired by popular locative media Apps such as Foursquare and Facebook Places. Users are familiar with user interfaces that allow them to check-in places and provide implicit (e.g., number or total check-ins) or direct feedback (e.g., stars or tags) about the respective locations. Locative media Apps employ very simple scoring systems, such as points for each check-in and badges for repeated check-ins, as well as stars and tags. Our user interface builds upon the above familiarity and enables the users to "check-in" routes instead of fixed places. Existing locative media Apps also provide a user interface for reporting bad content. Similarly, our user interface has successfully encouraged peer-reviewing of uploaded routes by introducing a simple scoring system. Further research is required for crowdsourcing and gamifying the manual effort that is necessary for the post-processing and the final cartographic visualization, since uploaded routes are not visually pleasing or accurate as such.

\section{Conclusion}

"A map is not the territory it represents, but, if correct, it has a similar structure to the territory, which accounts for its usefulness" Korzybski [7].

This work is based on the realization that an abstract map is more useful than a realistic one in many cases, as long as the abstraction is fit for the user task. In our cartography, we employ a different color shade, according to route type e.g. sidewalk, or pedestrian walkway, which is a common practice in mapmaking. For example, the width of roads should be illustrated according to the respective pedestrian popularity, which might vary according to seasons, time of the days, and personal preferences, which are worth exploring in further research. Moreover, further research should consider alternative cartographic visualizations (e.g., a popular route might fade in visibility if it is not being followed after a certain amount of time), as well as the needs of alternative users groups, such as cyclists and users with special needs.

Most notably, our work has emphasized the creation of a pedestrian map with pragmatic routes that have been followed by fellow city dwellers in the past. If citizens consulted a pedestrian-centered map and found that the respective path was considered walkable by many inhabitants, they would be motivated to switch the means of 
transportation and commute towards more walking, thus improving well-being. Pedestrian-centered cartography would also lead to a more walkable city, since city officials and activists could deploy plans based on pragmatic data: actual walkable (or not) paths. Overall, the result would be beneficial for the city and the quality of life would be increased [36]. In conclusion, we suggest that the App can be expanded easily for the creation of other types of maps, besides pedestrians', e.g., cyclists, accessibility. In addition to producing new kinds of cartography, we suggest that long-term civic engagement in the identification of walkable routes might increase well-being and quality of life in cities.

\section{Authors' contributions}

IK co-authored the article, co-designed the evaluation, co-designed the user interface, and implemented the system. KC co-authored the article, co-designed the evaluation, and co-designed the user interface. Both authors read and approved the final manuscript.

\section{Competing interests}

The authors declare that they have no competing interests.

Received: 26 August 2016 Accepted: 11 February 2017

Published online: 19 April 2017

\section{References}

1. Leslie E, Coffee N, Frank L, Owen N, Bauman A, Hugo G (2007) Walkability of local communities: using geographic information systems to objectively assess relevant environmental attributes. Health Place 13(1):111-122

2. Van Dyck D, Deforche B, Cardon G, De Bourdeaudhuij I (2009) Neighbourhood walkability and its particular importance for adults with a preference for passive transport. Health Place 15(2):496-504

3. Coulton P, Huck J, Gradinar A, Salinas L (2017) Mapping the beach beneath the street: digital cartography for the playable city. In: Playable cities. Springer, Singapore, pp 137-162

4. Chorianopoulos K (2014) Community-based pedestrian mapmaking. J Community Inform 10(3). http://ci-journal. net/index.php/ciej/article/view/1095. Accessed 18 Feb 2017.

5. Wilmott C (2016) Small moments in spatial big data: calculability, authority and interoperability in everyday mobile mapping. Big Data Soc 3(2):2053951716661364

6. Dent BD, Torguson JS, Hodler TW (1999) Cartography: thematic map design. McGraw, New York

7. Korzybski A (1996) [1st ed. 1933]. Chapter IV: on structure. In: Schuchardt read, charlotte. science and sanity: an introduction to non-aristotelian systems and general semantics. pp 58

8. Sulleyman A (2015) New London tube map shows how long it takes to walk, not ride a train. http://gizmodo.com/ new-london-tube-maps-shows-how-long-it-takes-to-walk-n-1741879484. Accessed 24 May 2016

9. Heipke C (2010) Crowdsourcing geospatial data. ISPRS J Photogramm Remote Sens 65(6):550-557

10. Haklay M (2010) How good is volunteered geographical information? A comparative study of openstreetmap and ordnance survey datasets. Environ Plan 37(4):682-703

11. Quercia D, Schifanella R, Aiello LM (2014) The shortest path to happiness: recommending beautiful, quiet, and happy routes in the city. In: Proceedings of the 25th ACM conference on hypertext and social media. ACM, New York, pp 116-125

12. Silvertown J (2009) A new dawn for citizen science. Trends Ecol Evol 24(9):467-471

13. Bonney R, Cooper CB, Dickinson J, Kelling S, Phillips T, Rosenberg KV, Shirk J (2009) Citizen science: a developing tool for expanding science knowledge and scientific literacy. Bioscience 59(11):977-984

14. Schlossberg M, Brehm C (2009) Participatory geographic information systems and active transportation: collecting data and creating change. Transp Res Rec J Transp Res Board 2105:83-91

15. Deterding S, Dixon D, Khaled R, Nacke L (2011) From game design elements to gamefulness: defining gamification. In: Proceedings of the 15th international academic MindTrek conference: envisioning future media environments. ACM, New York, pp 9-15

16. Smith H (2015) By the numbers: 17 important foursquare stats. http://expandedramblings.com/index.php/by-thenumbers-interesting-foursquare-user-stats/. Accessed 24 May 2016

17. Barkhuus L (2012) The mismeasurement of privacy: using contextual integrity to reconsider privacy in $\mathrm{HCl}$. In: Proceedings of the SIGCHI conference on human factors in computing systems. ACM, New York, pp 367-376

18. Cramer H, Rost M, Holmquist LE (2011) Performing a check-in: emerging practices, norms and 'conflicts' in locationsharing using foursquare. In: Proceedings of the 13th international conference on human computer interaction with mobile devices and services. ACM, New York, pp 57-66

19. Reddy S, Shilton K, Denisov G, Cenizal C, Estrin D, Srivastava M (2010) Biketastic: sensing and mapping for better biking. In: Proceedings of the SIGCHI conference on human factors in computing systems. ACM, New York, pp 1817-1820

20. Roccetti M, Marfia G, Palazzi CE (2011) Entertainment beyond divertissment: using computer games for city road accessibility. Comput Entertain 9(2):10 
21. Prandi C, Roccetti M, Salomoni P, Nisi V, Nunes NJ (2016) Fighting exclusion: a multimedia mobile app with zombies and maps as a medium for civic engagement and design. Multimed Tools Appl. 2016:1-29. doi:10.1007/s11042-0163780-9. Accessed 18 Feb 2017

22. Eickhoff C, Harris CG, de Vries AP, Srinivasan P (2012) Quality through flow and immersion: gamifying crowdsourced relevance assessments. In: Proceedings of the 35th international ACM SIGIR conference on research and development in information retrieval. ACM, New York, pp 871-880

23. Zuccarelli V (2012) How is stack overflow's reputation score calculated? https://www.quora.com/How-is-StackOverflows-reputation-score-calculated. Accessed 24 May 2016

24. Zandbergen PA (2009) Accuracy of iPhone locations: a comparison of assisted GPS, WiFi and cellular positioning. Trans GIS 13(s1):5-25

25. Kunic T (2013) Fused location provider. http://blog.lemberg.co.uk/fused-location-provider. Accessed 24 May 2016

26. Kasemsuppakorn P, Karimi HA (2013) A pedestrian network construction algorithm based on multiple GPS traces. Transp Res Part C Emerg Technol 26:285-300

27. Douglas DH, Peucker TK (1973) Algorithms for the reduction of the number of points required to represent a digitized line or its caricature. Cartogr Int J Geogr Inf Geovisualization 10(2):112-122

28. Williams M (2009) Google maps API tutorial: snap point to street. http://econym.org.uk/gmap/snap.htm. Accessed 24 May 2016

29. Miller $G$ (2014) The huge, unseen operation behind the accuracy of google maps. http://www.wired.com/2014/12/ google-maps-ground-truth/ Accessed 24 May 2016

30. Rossignol J (2016) iOS and android capture combine $98.4 \%$ share of smartphone market. http://www.macrumors. com/2016/02/18/ios-android-market-share-q4-15-gartner/. Accessed 24 May 2016

31. Kasemsuppakorn P, Karimi HA (2009) Pedestrian network data collection through location-based social networks, In: Collaborative computing: networking, applications and worksharing, 2009. 5th international conference on collaboratecom 2009. IEEE, New York, pp 1-9

32. Kumaraguru P, Cranor LF (2005) Privacy indexes: a survey of Westin's studies

33. Traunmueller M, Fatah gen Schieck A, Schöning J, Brumby DP (2013) The path is the reward: considering social networks to contribute to the pleasure of urban strolling. In: $\mathrm{CHI} 13$ extended abstracts on human factors in computing systems. ACM, New York, pp 919-924

34. Posti M, Schöning J, Häkkilä J (2014) Unexpected journeys with the HOBBIT: the design and evaluation of an asocial hiking app. In: Proceedings of the 2014 conference on designing interactive systems. ACM, New York, pp. 637-646

35. Haklay M, Basiouka S, Antoniou V, Ather A (2010) How many volunteers does it take to map an area well? The validity of Linus'law to volunteered geographic information. Cartogr J 47(4):315-322

36. Frank LD, Sallis JF, Conway TL, Chapman JE, Saelens BE, Bachman W (2006) Many pathways from land use to health: associations between neighborhood walkability and active transportation, body mass index, and air quality. J Am Plan Assoc 72(1):75-87

\section{Submit your manuscript to a SpringerOpen ${ }^{\circ}$ journal and benefit from:}

- Convenient online submission

- Rigorous peer review

- Immediate publication on acceptance

- Open access: articles freely available online

- High visibility within the field

- Retaining the copyright to your article

Submit your next manuscript at $\boldsymbol{\nabla}$ springeropen.com 